\title{
El control intensivo de la glucemia en diabéticos tipo 1 previene más complicaciones microvasculares si se logra precozmente
}

Effect of Intensive Therapy on the Microvascular Complications of Type 1 Diabetes Mellitus. The Writing Team for the Diabetes Control and Complications Trial/Epidemiology of Diabetes Interventions and Complications Research Group. JAMA. 2002 May 15; $287: 2563-2569$.

\section{Objetivo}

Evaluar el efecto a largo plazo del tratamiento intensivo de la glucemia en diabéticos tipo 1.

\section{Diseño}

Cohorte prospectiva con siete años de seguimiento luego de la finalización de un ensayo clínico aleatorizado.

\section{Lugar}

Estudio multicéntrico en EE.UU.

\section{Pacientes}

Pacientes diabéticos tipo 1 que participaron en el DCCT. Se incluyeron 1375 pacientes (95\% de los que terminaron el DCCT). De estos, la mitad pertenecían originalmente a la rama tratamiento intensivo y la otra mitad, a la de tratamiento convencional.

\section{Intervención}

Al finalizar el DCCT y vistos los beneficios del tratamiento intensivo con insulina, se indicó el mismo (tres o cuatro inyecciones diarias o bomba de infusión más monitoreo en sangre cuatro veces por día) a todos los pacientes.

\section{Medición de los resultados principales}

El análisis fue hecho por intención de tratar. Se midió la progresión de complicaciones microvasculares (retino y nefropatía) en los que habían pertenecido originalmente al grupo de tratamiento intensivo y a los que habían pertenecido al grupo de tratamiento convencional.

\section{Resultados}

Al final del primer año de seguimiento, $95 \%$ de los pacientes con tratamiento intensivo en DCCT usaban esta modalidad de tratamiento vs un $75 \%$ de los que originalmente tenían tratamiento convencional. Las hemoglobinas glicosiladas eran de $7.9 \%$ y $8.3 \%$ respectivamente. Esta diferencia de hemoglobinas glicosiladas fue disminuyendo entre ambos grupos y los valores promedio a lo largo de todo el seguimiento fueron de $8.3 \%$ para el grupo que originalmente tenía tratamiento convencional y $8.1 \%$ para el intensivo. A los siete años un $83 \%$ de los pacientes que en el DCCT tenían tratamiento convencional, recibían tratamiento intensivo. Comparando el grupo originalmente asignado a tratamiento intensivo con el originalmente asignado a tratamiento convencional, el OR para progresión de retinopatía fue de 0.35 (IC 95\% 0.16 a $0.74)$. Los pacientes que originalmente tenían tratamiento intensivo requirieron menos fotocoagulación con láser OR 0.23 (IC 95\%: $0.09-0.55)$.

El desarrollo de microalbuminuria y proteinuria en aquellos que no la presentaban al término del DCCT, fue reducida en un 53 y un $86 \%$ respectivamente.

Presentaron un resultado combinado que incluyó creatinina mayor a $2 \mathrm{mg} / \mathrm{dl}$ o diálisis crónica o transplante renal seis pacientes del grupo originalmente de tratamiento intensivo vs 17 del originalmente convencional .

\section{Conclusiones}

El efecto nocivo de la hiperglucemia en las complicaciones microvasculares de la dibetes tipo 1 persiste por un tiempo considerable luego del descenso de la glucemia. Los efectos beneficiosos del control estricto permanecen más allá del período de intervención más intensivo.

\section{Comentario}

El presente estudio reporta los resultados del seguimiento de la cohorte que se incluyó en el DCCT. EI DCCT' había demostrado por primera vez en un ensayo aleatorizado que el control estricto de la glucemia prevenía o al menos retrasaba la aparición de complicaciones microvasculares en diabéticos tipo 1. EI DCCT tuvo que ser interrumpido prematuramente luego de 6.5 años de seguimiento. Esos mismos pacientes participaron de esta fase del estudio en la cual todos fueron estimulados a recibir tratamiento intensivo. Si bien las hemoglobinas glicosiladas no difirieron de manera importante entre los pacientes que en el DCCT estaban bajo tratamiento intensivo y los que recibían tratamiento convencional, el presente trabajo muestra que esos años durante los cuales un grupo tuvo control más estricto que otro no pueden "recuperarse" en términos de reducción de complicaciones. Estos hallazgos son consistentes con lo observado en el DCCT ya que en dicho estudio, el nivel basal de hemoglobina glicosilada fue identificado como factor de riesgo para futuras complicaciones. Más aun, si bien la mayor diferencia de hemoglobinas gli- cosiladas entre las ramas del DCCT se logró a los seis meses luego de la aleatorización, hicieron falta entre tres y cuatro años de tratamiento para evidenciar las diferencias en la incidencia acumulada de retinopatía o nefropatía.

Los resultados del presente estudio sugieren que la hiperglucemia tiene efectos crónicos y a largo plazo sobre la fisiopatología subyacente al desarrollo de las complicaciones y no agudos. Lleva bastante tiempo de mejora en el control metabólico para contrarrestar el efecto de hiperglucemias prolongadas. Una vez que esto sucede, el beneficio es duradero.

Conclusion del comentador: En diabéticos tipo I, el control precoz de la glucemia se asocia a menor riesgo de desarrollo de nefropatía y retinopatía. Estos resultados deben estimular a los médicos y pacientes a lograr un control estricto de la glucemia lo más precoz y duradero en el tiempo como sea posible.

Dra. Karin Kopitowski [ Unidad de Medicina familiar y Preventiva. Hospital Italiano de Buenos Aires ] 